\title{
Structure of a New Dense Amorphous Ice
}

\author{
J. L. Finney, ${ }^{1}$ D. T. Bowron, ${ }^{1,2}$ A. K. Soper, ${ }^{1,2}$ T. Loerting, ${ }^{3}$ E. Mayer, ${ }^{3}$ and A. Hallbrucker ${ }^{3}$ \\ ${ }^{1}$ Department of Physics and Astronomy, University College London, Gower Street, London WC1E 6BT, United Kingdom \\ ${ }^{2}$ ISIS Facility, Rutherford Appleton Laboratory, Chilton, Didcot, Oxon OX11 OQX, United Kingdom \\ ${ }^{3}$ Institut für Allgemeine und Anorganische Chemie, Universität Innsbruck, Innrain 52a, A-6020 Innsbruck, Austria
}

(Received 11 July 2002; published 29 October 2002)

\begin{abstract}
The detailed structure of a new dense amorphous ice, VHDA, is determined by isotope substitution neutron diffraction. Its structure is characterized by a doubled occupancy of the stabilizing interstitial location that was found in high density amorphous ice, HDA. As would be expected for a thermally activated unlocking of the stabilizing "interstitial," the transition from VHDA to LDA (low-density amorphous ice) is very sharp. Although its higher density makes VHDA a better candidate than HDA for a physical manifestation of the second putative liquid phase of water, as for the HDA case, the VHDA to LDA transition also appears to be kinetically controlled.
\end{abstract}

DOI: $10.1103 /$ PhysRevLett.89.205503

High-density amorphous ice (HDA) and the apparently first-order transition between HDA and low-density amorphous ice (LDA) play key roles in the concept of polyamorphism of one-component systems [1,2] and the liquid-liquid phase transition hypothesis where a second critical point at low temperature is assumed to cause the anomalous properties of supercooled water [3-6] (for recent reviews, see [7,8]). Debenedetti [7] emphasized that "the relationship between LDA and HDA is of fundamental importance, not just for understanding the phase behavior of water, but for the physics of disordered systems in general." HDA was first made by Mishima et al. $[1,2]$ by compression of hexagonal ice (ice $\mathrm{I} h$ ) at $77 \mathrm{~K}$ and $1.0 \mathrm{GPa}$, and the HDA-LDA transition has been characterized by many techniques $[1,2,9-20]$. HDA has been considered to be a glassy state of high-pressure liquid water $[1,2,14,15,21,22]$ or a collapsed poorly crystalline phase $[23,24]$, and discussions about these interpretations continue (reviewed in Refs. [7,8]). Its structure has been argued to be similar to that of high-pressure liquid water which suggests that it is a glassy form of the latter [8], but its glass transition has not so far been observed.

Very recently it has been shown that a second distinct structural form of high-density amorphous ice can be recovered at $77 \mathrm{~K}$ and 1 bar, after isobaric heating of HDA under pressure [25]. Named "very-high density amorphous ice" (VHDA), this form has been characterized by $\mathrm{x}$-ray diffraction, with the main diffraction peak [25] being both sharpened and shifted to a higher scattering vector compared to HDA [1,2,15,21], while the Raman spectra of HDA and VHDA exhibit pronounced differences in frequencies of the coupled $\mathrm{OH}$, decoupled $\mathrm{OD}$, and librational and translational bands $[25,26]$. The density of VHDA is $1.25 \pm 0.01 \mathrm{~g} \mathrm{~cm}^{-1}$ [25], some 7\%$8 \%$ higher than that of HDA [1,2]. On heating VHDA to $\approx 140 \mathrm{~K}$ at a starting pressure of $0.02 \mathrm{GPa}$ under isochoric conditions, HDA is regained, whereas isobaric heating at low pressures, e.g., at $0.11 \mathrm{GPa}$, to $\approx 127 \mathrm{~K}$
PACS numbers: 61.43.Fs, 61.12.-q

leads directly to the formation of LDA, without intermediate formation of HDA [25].

As this new ice appears to be a distinct, new amorphous form that is kinetically stable under ambient pressure, its structure - and its relationship to known forms - is of intense interest in the context of the phase behavior of metastable water and of the controversial hypothesis of the existence of two critical points in water [8]. We report here the determination of its detailed structure.

VHDA samples were prepared in principle as described in Ref. [25]. As relatively large samples were required, a $2.0 \mathrm{~cm}$ inner diameter piston cylinder apparatus and a 200 ton hydraulic press were used instead of the equipment used for the studies of Ref. [25]. Two of each $\mathrm{D}_{2} \mathrm{O}$, $\mathrm{HDO}$, and $\mathrm{H}_{2} \mathrm{O}$ samples were prepared by filling $3.00 \mathrm{ml}$ of the liquid into the precooled $2.0 \mathrm{~cm}$ inner diameter piston cylinder apparatus. The cylinder was prelined with a $0.5 \mathrm{~mm}$ thick Indium foil to avoid pressure drops during the initial compression cycle of HDA formation $[1,27]$. With the arrangement immersed in liquid nitrogen the pressure was increased to $1.34 \mathrm{GPa}$ for $\mathrm{H}_{2} \mathrm{O}, 1.44 \mathrm{GPa}$ for $\mathrm{HDO}$, and $1.50 \mathrm{GPa}$ for $\mathrm{D}_{2} \mathrm{O}$, respectively, within 12 to $16 \mathrm{~min}$. The pressure was then reduced to $\approx 1.15 \mathrm{GPa}$, the coolant pumped off, and the sample allowed to warm to $160 \mathrm{~K}\left(\mathrm{H}_{2} \mathrm{O}\right), 163 \mathrm{~K}(\mathrm{HOD})$, or $166 \mathrm{~K}\left(\mathrm{D}_{2} \mathrm{O}\right)$, with the pressure maintained constant to within $\pm 5 \%$, over periods of about 50 to $60 \mathrm{~min}$. The mold was cooled subsequently by pouring liquid nitrogen into the containing vessel and the sample was recovered at $77 \mathrm{~K}$ and ambient pressure. X-ray diffraction confirmed these samples were indistinguishable from those reported in [25] and also that the isotopically distinct samples were structurally indistinguishable. IR spectroscopy gave $99.0 \mathrm{~mol} \%$ and $49.7 \mathrm{~mol} \%$ for deuterium concentrations of the $\mathrm{D}_{2} \mathrm{O}$ and HDO samples, respectively.

Each sample was loaded under liquid nitrogen into a parallel sided TiZr cell for data collection at $80 \mathrm{~K}$ on the SANDALS diffractometer of the ISIS pulsed spallation neutron source, UK. The measured neutron diffraction 
patterns showed no sign of Bragg peaks that would have indicated the presence of crystalline material. After data collection on VHDA, the temperature of each sample was raised to $136 \mathrm{~K}$ for the in situ conversion to what was expected to be LDA. The temperature was then reduced and further data taken at $80 \mathrm{~K}$. Background, can scattering, multiple scattering, and attenuation corrections were made using the ATLAS data analysis suite [28]. The data were then normalized for powder packing fraction as described in Ref. [9]. Subsequent spectroscopic analysis of the samples confirmed the isotopic compositions of the $\mathrm{D}_{2} \mathrm{O}$ and 50:50 $\mathrm{H}_{2} \mathrm{O} / \mathrm{D}_{2} \mathrm{O}$ samples were unchanged.

In order to allow exploration of the geometries of the intermolecular structures consistent with the measured partial pair correlation functions, the empirical potential structure refinement (EPSR) procedure [29] was implemented [9]. This process performs a Monte Carlo computer simulation of the system under study to generate ensembles of water molecules whose structures are consistent with the measured diffraction data. The water molecule ensembles so obtained are used to calculate structural quantities such as site-site radial distribution functions (rdfs, Fig. 1) and spatial density functions (sdfs, Fig. 2). For the respective systems VHDA and LDA, the atomic densities used within the structure refinements were 0.125 and 0.0937 atoms $\AA^{-3}$. The similarity with those of LDA [9] of the partial rdfs obtained after the transformation (not shown) confirms that the transformed material was indeed LDA.

Comparing first the $\mathrm{OH}$ and $\mathrm{HH}$ partial rdfs of VHDA with those obtained earlier [9] on HDA (Fig. 1), these functions for the two structures are similar. The slight inward shift of the first maximum in the $\mathrm{OH}$ rdf may indicate a slight shortening of the hydrogen bond in VHDA. The first $\mathrm{OH}$ coordination number for VHDA of $2.0(0.1)$ in the range from $1.4 \AA$ to $2.5 \AA$ indicates that each water molecule makes on average two hydrogen bonds, as is the case for HDA [9]. We conclude that in VHDA, as well as HDA, we have a tetrahedrally coordinated fully hydrogen bonded network.

In contrast, the OO rdf for VHDA shows major changes from that of HDA. The broad, incipiently split second peak in HDA appears to shift inwards to form a shoulder on the first peak. Integrating under this first peak out to the first minimum for HDA ( $2.3 \AA$ to $3.3 \AA$ ) shows that the number of neighbors out to this distance has increased from the 5.0(0.1) of HDA to almost 6, [5.8(0.1)] for VHDA.

In HDA, it has been argued that this additional first neighbor "interstitial" molecule acts to stabilize the HDA structure by some kind of "pinning" mechanism. The interesting question then arises as to the location and possibly related function in VHDA of this additional sixth molecule in the first neighbor shell.

We consider first the coordination number calculated out to the first minimum in the OO partial rdf of VHDA which is at 3.1 $\AA$. Interestingly, we find a value of essen-

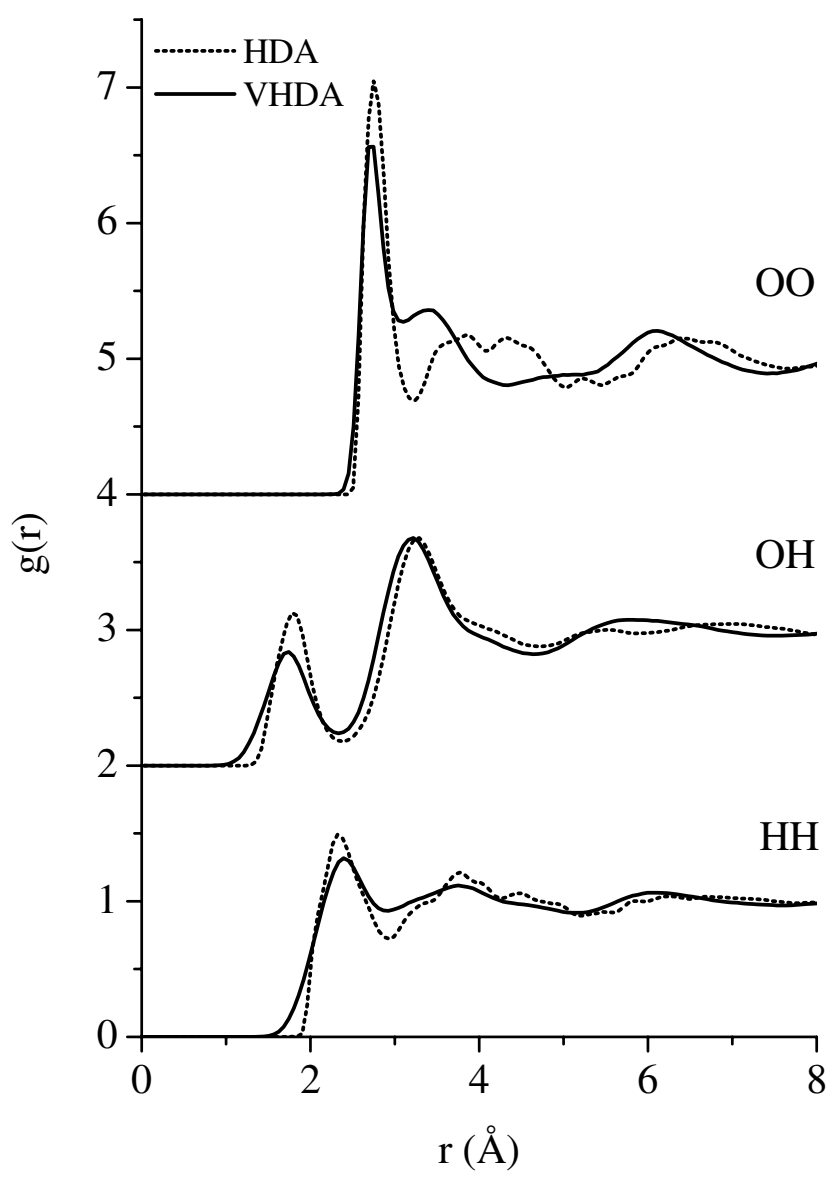

FIG. 1. Intermolecular partial radial distribution functions of VHDA and HDA at $80 \mathrm{~K}$. For clarity, the ordinates for $g_{\mathrm{OH}}(r)$ and $g_{\mathrm{OO}}(r)$ are shifted by 2 and 4 , respectively.

tially 4 [4.1(0.1)] for both HDA and VHDA. The obvious inference is that these are the four tetrahedrally coordinated waters hydrogen bonded to the central molecule, an inference confirmed by the sdf taken out to this distance [see the first pair of frames in Fig. 2(a)]: for both VHDA and HDA we see the classical water environment of two lobes above the central molecule relating to the two neighbors acting as proton acceptors, with the lobe(s) beneath corresponding to the two waters donating protons to the lone pair region of the central molecule. The fact that the lower lobe is separated into two for HDA is a consequence of the level at which the sdf is plotted, which is set to emphasize the dominant directions of the neighbor interactions. That the VHDA sdf retains a broader single lobe is suggestive of a slightly greater directional smearing of the first shell neighborhood of the lone pairs that is presumably a consequence of the higher density.

Moving to the second pair of frames in Fig. 2(b), which shows the spatial distribution of water neighbors between 3.1 and $3.3 \AA$ (the minimum in the HDA OO partial rdf [9]), we see a strong similarity between VHDA and HDA: the two large lobes are those of the interstitial "lynch pin" molecule that was identified previously in HDA [9]. However, we deduce from the coordination numbers that 
(a)
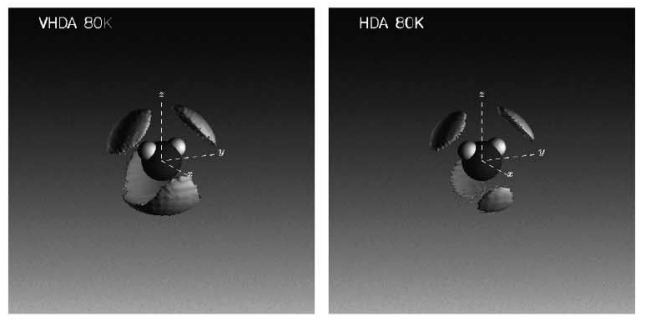

(b)
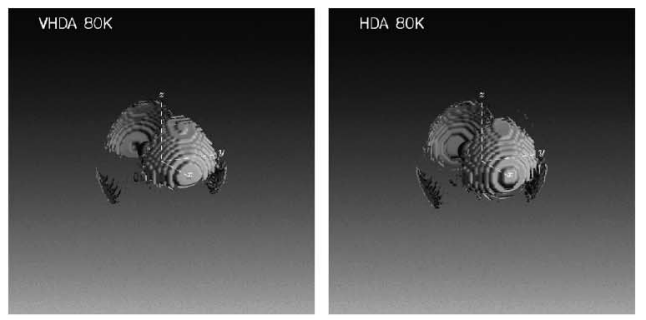

(c)
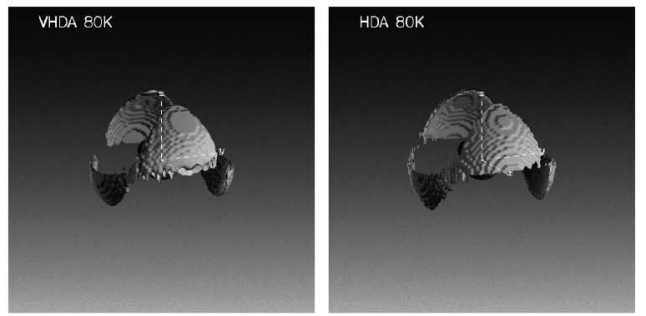

(d)
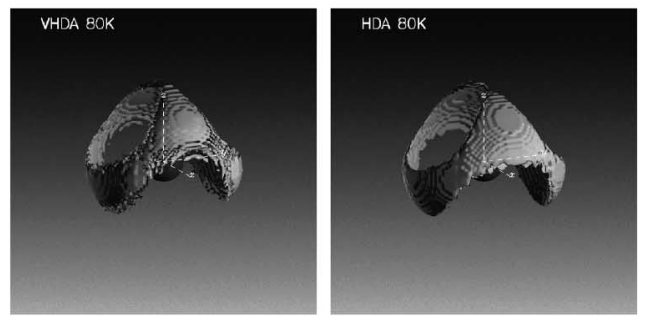

FIG. 2. Spatial density functions showing the distribution of water molecules in shells of increasing distance around a central water molecule for VHDA (left panels) and HDA (right panels) at $80 \mathrm{~K}$. The HDA data are from the work in Ref. [9]. The contour levels are chosen such that $30 \%$ of the molecules are included in the lobes, which therefore indicate the main orientational arrangements of the waters in the shells. The shell boundaries are chosen to relate to relevant features in the $g_{\mathrm{OO}}(r) \mathrm{s}$ of both structures (Fig. 1): (a) $2.3 \AA-3.1 \AA$, (b) $3.1 \AA-3.3 \AA$, (c) $3.3 \AA-3.7 \AA$, and (d) $3.7 \AA-4.1 \AA$. The coordination numbers that are associated with each of these panels are (a) 4.1(0.1) for both VHDA and HDA, (b) 1.7(0.1) and $0.9(0.1)$ for VHDA and HDA, respectively, (c) 3.5(0.1) and 2.2(0.1) for VHDA and HDA, and last (d) 2.7(0.1) and 3.5(0.1) for VHDA and HDA. This gives a total occupancy for the full second neighbor shell (b) $+(\mathrm{c})+(\mathrm{d})$ of $7.9(0.1)$ molecules for VHDA and 6.6(0.1) for HDA. The viewport for each panel is set to $15 \AA \times 15 \AA$, with the water oxygen at the center.

there are approximately two molecules associated with these lobes in VHDA compared to the one in HDA. Thus, in going from HDA to VHDA, an additional molecule has been forced into the lynch pin location. In HDA, one water would be distributed between the two lobes, giving effectively $50 \%$ occupancy of each. In VHDA, we have forced an increase to essentially $100 \%$ occupancy of each lynch pin position.

Finally, farther out from the central molecule, in moving from HDA to VHDA the OO rdf of Fig. 1 suggests molecules are pushed inwards from the 3.7-4.1 $\AA$ region to the 3.3-3.7 $\AA$ interval. That this inward shift reflects the consequence of a simple compression-i.e., without significant orientational reorganization-is implied by the similarities between the sdfs for these two respective shells as shown in Figs. 2(c) and 2(d). Both shells in VHDA and HDA have the characteristic spatial structure of the water second neighbor shell. The only difference is that in going from HDA to VHDA, approximately one molecule shifts from the outer to the inner of these two shells.

A further point is evident from plotting the sdfs in sequential shells rather than in spheres of increasing radii as was done in an earlier publication on HDA and LDA [9]. The lynch pin lobes in the 3.1-3.3 $\AA$ shell are now seen to be contiguous with the normal second neighbor shell. This is consistent with an interpretation of the lynch pin molecules in VHDA arising from molecules in the second shell being pushed closer towards the central molecule. In light of this, we begin to see how the structure of VHDA is obtained by a simple compressive distortion of the HDA structure. Moreover, a similar comment can be made about how the structure of HDA is obtained from that of liquid water. The results presented here thus imply that the structures of liquid water, HDA and VHDA, bear a simple relationship to each other without the need to postulate any significant structural reorganization. We move from liquid water through HDA to VHDA through the progressive increase in occupancy of the lynch pin interstitial site from about $20 \%$ (corresponding to the experimental coordination of 4.4) in liquid water, through $50 \%$ in HDA to $~ 100 \%$ in VHDA. We might therefore tentatively postulate that VHDA represents the limit in density without the need to force a structural reorganization, for example, through the creation of partially interpenetrating networks as seen in the higher pressure ice structures [30].

The transition at ambient pressure from VHDA to LDA occurs at about $127 \mathrm{~K}$ at a heating rate of $10 \mathrm{~K} \mathrm{~min}^{-1}$. This temperature is some $14 \mathrm{~K}$ higher than the HDA to LDA transformation measured in [13]. Although some of this difference is due to differences in measuring conditions, the transformation temperature of VHDA is clearly significantly higher than that of HDA. Moreover, this transition is calorimetrically very sharp (A. Hallbrucker, unpublished results), with no sign of any lower temperature feature such as that observed by Handa et al. [13] for the HDA to LDA transition. The sharpness of this process is consistent with the VHDA to LDA transition being kinetically constrained at low temperature, where insufficient molecular kinetic energy is available to enable the interstitial molecules to jump out of their first shell positions. With a higher occupancy of 
this interstitial location in VHDA, the locking up of the structure is likely to be greater than for HDA, requiring additional kinetic energy to unlock it, hence the significantly higher transition temperature of VHDA. The specific mechanism by which this interstitial stabilizes the VHDA and HDA structures remains to be determined.

Very high-density amorphous ice is a material that can, like HDA, be recovered to ambient pressure at liquid nitrogen temperature. As for HDA [9], there is no evidence in the diffraction pattern that it is a microcrystalline rather than a genuinely amorphous structure. It can thus be regarded as a fifth amorphous form of water which is stable at low temperature under ambient pressure conditions. In the two-liquid scenario of water, it raises the possibility that VHDA rather than HDA might be better regarded as a physical manifestation of this second putative liquid phase. The similarity between the $\mathrm{OO}$ functions for VHDA and the high-density water of Ref. [31] is also suggesting in this respect. The structure of VHDA although distinct, can be related to that of HDA by a doubled occupancy of the interstitial molecule located in a shell just $0.1-0.2 \AA$ more distant from a typical central molecule than the directly hydrogen bonded neighbors. Like HDA and LDA, VHDA is also a fully hydrogen bonded tetrahedral network structure. The interstitial site is seen to be a "pushed inwards" part of the normal second neighbor shell, and thus there is a clear structural relationship from liquid water through HDA to VHDA which is characterized by an increasing occupancy of the interstitial site. We might postulate that there is a degree of occupancy of this site at which the structure "locks in" to one which is stable at low temperature. There is no need to postulate any significant reorganization of the network structure in moving between these structures - on the basis of the data presented here, they appear to be topologically isomorphous. Though in this context the interstitial occupancy might be thought of as some sort of order parameter for the water system, there is no evidence that intermediate structures with intermediate values of this parameter can be made stably at ambient pressure (though they may be observable as the pressure on HDA is continuously increased). Understanding why these two specific occupancies stabilize the two respective structures (HDA and VHDA) may suggest the specific mechanism by which the interstitial lynch pin stabilizes these structures.

Finally, the transition from VHDA to LDA is very sharp, as would be expected for an activated unlocking of the interstitial molecules that stabilize the structure. These results are consistent with the VHDA to LDA transition being kinetically inhibited.

We thank the ISIS Facility for neutron beam time and support facilities.
[1] O. Mishima, L. D. Calvert, and E. Whalley, Nature (London) 310, 393 (1984).

[2] O. Mishima, L. D. Calvert, and E. Whalley, Nature (London) 314, 76 (1985).

[3] P. H. Poole, F. Sciortino, U. Essman, and H. E. Stanley, Nature (London) 360, 324 (1992).

[4] P. H. Poole, F. Sciortino, U. Essman, and H. E. Stanley, Phys. Rev. E 48, 3799 (1993).

[5] H. E. Stanley, C. A. Angell, U. Essman, M. Hemmati, P. H. Poole, and F. Sciortino, Physica (Amsterdam) 206A, 1 (1994).

[6] P. H. Poole, F. Sciortino, T. Grande, H. E. Stanley, and C. A. Angell, Phys. Rev. Lett. 73, 1632 (1994).

[7] P.G. Debenedetti, Metastable Liquids (Princeton University Press, Princeton, NJ, 1996).

[8] O. Mishima and H. E. Stanley, Nature (London) 396, 329 (1998).

[9] J. L. Finney, A. Hallbrucker, I. Kohl, A. K. Soper, and D. T. Bowron, Phys. Rev. Lett. 88, 225503 (2002).

[10] L. Bosio, G. P. Johari, and J. Teixeira, Phys. Rev. Lett. 56, 460 (1986).

[11] A. Bizid, L. Bosio, A. Defrain, and M. Oumezzine, J. Chem. Phys. 87, 2225 (1987).

[12] M.-C. Bellissent-Funel, J. Teixeira, and L. Bosio, J. Chem. Phys. 87, 2231 (1987).

[13] Y. P. Handa, O. Mishima, and E. Whalley, J. Chem. Phys. 84, 2766 (1986).

[14] M. A. Floriano, Y. P. Handa, D. D. Klug, and E. Whalley, J. Chem. Phys. 91, 7187 (1989).

[15] O. Mishima, J. Chem. Phys. 100, 5910 (1994).

[16] D. D. Klug, O. Mishima, and E. Whalley, J. Chem. Phys. 86, 5323 (1987).

[17] E. Whalley, J. Less-Common Met. 140, 361 (1988).

[18] H. Kanno, K. Tomikawa, and O. Mishima, Chem. Phys. Lett. 293, 412 (1998).

[19] Y. Suzuki, Y. Takasaki, Y. Tominaga, and O. Mishima, Chem. Phys. Lett. 319, 81 (2000).

[20] O. Mishima, Science 254, 406 (1991).

[21] O. Mishima, Nature (London) 384, 546 (1996).

[22] E. Whalley, D. D. Klug, and Y. P. Handa, Nature (London) 342, 782 (1989).

[23] J. S. Tse, J. Chem. Phys. 96, 5482 (1992).

[24] J. S. Tse, D. D. Klug, C. A. Tulk, I. Swainson, E. C. Svensson, C.-K. Loong, V. Shpakov, V. R. Belosludov, R.V. Belosludov, and Y. Kawazoe, Nature (London) 400, 647 (1999).

[25] T. Loerting, C. Salzmann, I. Kohl, E. Mayer, and A. Hallbrucker, Phys. Chem. Chem. Phys. 3, 5355 (2001).

[26] C. A. Tulk, D. D. Klug, R. Branderhorst, P. Sharpe, and J. A. Ripmeester, J. Chem. Phys. 109, 8478 (1998).

[27] I. Kohl, E. Mayer, and A. Hallbrucker, Phys. Chem. Chem. Phys. 3, 602 (2001).

[28] A. K. Soper, W. S. Howells, and A. C. Hannon, Report No. RAL-89-046, 1989.

[29] A. K. Soper, Chem. Phys. 202, 295 (1996).

[30] W. F. Kuhs, J. L. Finney, C. Vettier, and D.V. Bliss, J. Chem. Phys. 81, 3612 (1984).

[31] A. K. Soper and M. A. Ricci, Phys. Rev. Lett. 84, 2881 (2000). 